\title{
REVISÃO SISTEMÁTICA SOBRE MÉTODOS DE DETERMINAÇÃO DE GASTO E CONSUMO ENERGÉTICOS EM CRIANÇAS E ADOLESCENTES
}

\author{
SYSTEMATIC REVIEW ABOUT METHODS OF ENERGY EXPENDITURE AND \\ ENERGY INTAKE IN CHILDREN AND ADOLESCENTS
}

\begin{abstract}
RESUMO
O objetivo dessa revisão sistemática foi identificar, descrever e analisar os métodos de determinação do gasto e consumo energéticos em estudos epidemiológicos clínicos e populacionais. A população do estudo foi de crianças e adolescentes na faixa etária de 6 a 18 anos. Para isso, foi realizada uma busca na base de dados Medline. Foram encontrados 141 artigos, dos quais 32 foram selecionados. Desses, foram obtidos 10 artigos, sendo 6 estudos de epidemiologia clínica e 4 de epidemiologia populacional. Nos estudos de epidemiologia clínica, os métodos usados para a determinação do gasto energético foram água duplamente marcada e calorimetria indireta. Nos estudos de epidemiologia populacional, o questionário de atividade física foi utilizado para determinação do gasto energético com atividade física. Os métodos de avaliação do consumo alimentar utilizados, tanto em epidemiologia clínica quanto em populacional, foram recordatório de 24 horas, registro alimentar e questionário de freqüência alimentar. São discutidas questões como a forma de realização dos métodos, a validação e adaptações para a população de crianças e adolescentes. Conclui-se que cuidados metodológicos devem ter maior apreço no design dos estudos epidemiológicos, pois podem enviesar os resultados e limitar conclusões.
\end{abstract}

Palavras-chave: Gasto energético; Consumo energético; Métodos; Epidemiologia; Crianças; Adolescentes.

\section{ABSTRACT}

The objective of this systematic review was to identify, describe and analyze methods used for determining energy expenditure and energy intake in clinical and population-based epidemiological studies. The study population was made up of children and adolescents aged from 6 to 18 years. The articles were identified by means of searching the Medline database. A total of 141 articles were identified, 32 of which were selected. From these, 10 articles were chosen, 6 of which were clinical epidemiological studies and 4 were populational epidemiological studies. The methods used to determine energy expenditure in the clinical studies were doubly labeled water and indirect calorimetry. In the populational studies, questionnaires on physical activity were used to determine the energy expended during physical activity. The methods used to evaluate nutritional intake, both in the clinical and populational epidemiological studies, were 24-hour dietary recalls, food records and food frequency questionnaires. The review goes on to discuss questions such as the manner in which these methods are employed and their validation and adaptation for populations of children and adolescents. It is concluded that care must be taken with the methodological aspects of epidemiological study design in order to avoid introducing bias to results and limiting conclusions.

Key words: Energy expenditure; Energy intake; Methods; Epidemiology; Children; Adolescents.

1 Instituto de Saúde Coletiva da Universidade Federal da Bahia. Brasil.

2 Faculdade de Saúde Pública da Universidade de São Paulo. São Paulo. Brasil

3 Programa de Pós-Graduação em Nutrição. Departamento de Nutrição. Centro de Ciências da Saúde. Universidade

Federal de Santa Catarina. Florianópolis, SC, Brasil. 


\section{INTRODUÇÃO}

Nas duas últimas décadas, houve um crescente interesse no meio científico em relação às doenças crônicas não transmissíveis e suas conseqüências biológicas e sociais. A obesidade tornou-se problema de saúde pública, em decorrência tanto do aumento da sua prevalência em diversos países, principalmente nos Estados Unidos da América, como da sua forte associação com doenças crônicas não transmissíveis ${ }^{1-3}$.

A obesidade pode ser considerada um problema de desequilíbrio no balanço energético. O balanço energético é determinado pela relação entre gasto e consumo energéticos. Quando o gasto calórico é maior que o consumo de energia, o suprimento energético é mantido através da depleção de tecidos internos (principalmente gorduras e proteínas). Quando o consumo energético é maior que o gasto, há o acúmulo de tecidos internos (principalmente gordura na forma de tecido adiposo). Isso pode ser demonstrado pela equação: consumo energético = gasto energético + armazenamento energético ${ }^{4}$.

Na verdade, o balanço energético é a maquinaria fisiológica que regula o peso corporal, armazenamento de energia e depósito de tecidos, determinando também o consumo e gasto energéticos. Aspectos como temperatura, hipóxia (baixo nível de $\mathrm{O}_{2}$ ), infecções, doenças, traumas, gravidez, crescimento e desenvolvimento, tamanho corporal, atividade física e ingestão de alimentos podem ser determinantes do balanço energético ${ }^{5,6}$.

Para Speakman 4 , a obesidade é decorrente da interrelação entre genética e ambiente, o que determina a fisiologia e o comportamento de indivíduos e tem influência sobre o balanço energético. Portanto, a obesidade ocorre quando o desequilíbrio no balanço energético permanece por um longo período de tempo, sem que haja alternância de períodos com mobilização energética ${ }^{6}$.

Nos estudos que tentam averiguar a etiologia da obesidade, medidas de gasto e consumo energéticos são amplamente utilizadas.

A população adulta de homens e mulheres foi muito pesquisada em relação à determinação de gasto e consumo energéticos, o que acarretou num grande número de métodos para tal população. Mais recentemente, entretanto, tem aumentado o número de publicações com a população de crianças e adolescentes, haja vista o interesse na etiologia de doenças, como a obesidade, e na formulação de políticas públicas ${ }^{1,3,7}$.

Os problemas com as medidas de gasto e consumo energéticos utilizados com a população de crianças e adolescentes, são agravados devido ao fato de ainda haver pouca informação dos métodos possíveis de se utilizar, quando comparados com os adultos $^{8}$. É sabido que a infância e a adolescência são períodos de um intenso processo de crescimento e desenvolvimento que produz alterações significativas em características de ordem física, como nos componentes corporais, bem como em características de ordem cognitiva, social e afetiva ${ }^{9}$. Assim, estudos epidemiológicos de obesidade podem ter sua força contestada frente a aspectos como falta de validade ou de adaptações desses métodos para a população de crianças e adolescentes ${ }^{10,11}$.

Considerando a relevância do tema e o crescente interesse nessa área, o objetivo desse estudo foi identificar, descrever e analisar os métodos de determinação do gasto e consumo energético, em pesquisas epidemiológicas clínicas e populacionais, realizadas na população de crianças e adolescentes dos 6 aos 18 anos de idade, nos últimos 10 anos.

\section{PROCEDIMENTOS METODOLÓGICOS}

Realizou-se revisão sistemática, utilizando a base eletrônica de dados da National Library of Medicine (MEDLINE). Os unitermos empregados foram energy expenditure, energy intake, methods e epidemiological study. Os limites ajustados no MEDLINE contemplaram humanos, crianças (6-12 anos), adolescentes (12-18 anos) e artigos publicados nos últimos 10 anos. A busca na base de dados foi realizada no mês de abril de 2006 .

Foram encontrados 141 estudos com as condições acima consideradas. Para definir quais desses fariam

Quadro 1. Tipo do estudo, método de mensuração e freqüência dos estudos não elegíveis e elegíveis na revisão.

\begin{tabular}{|c|c|c|c|c|}
\hline $\begin{array}{c}\text { Não Elegíveis e } \\
\text { Elegíveis }\end{array}$ & Tipo do Estudo & Mensuração & $\mathrm{n}$ & $\%$ * \\
\hline \multirow{5}{*}{$\begin{array}{c}\text { Estudos } \\
\text { Não Elegíveis }\end{array}$} & Não Epidemiológico & Gasto e/ou Consumo Energético & 43 & 30,5 \\
\hline & Não Epidemiológico & $\begin{array}{l}\text { Não mensura gasto elou consumo } \\
\text { energético (total ou quantitativa- } \\
\text { mente) }\end{array}$ & 19 & 13,5 \\
\hline & Validação & - & 19 & 13,5 \\
\hline & Revisão & - & 10 & 7,1 \\
\hline & Com Adultos ou Crianças $<6$ anos & - & 18 & 12,8 \\
\hline & \multicolumn{2}{|r|}{ Sub-Total } & 109 & 77,3 \\
\hline \multirow{8}{*}{ Estudos Elegíveis } & Epidemiológico clínico & Gasto Energético & 02 & 1,4 \\
\hline & & Consumo Energético & 07 & 5,0 \\
\hline & & Ambos & 19 & 13,5 \\
\hline & Epidemiológico populacional & Gasto Energético & 01 & 0,7 \\
\hline & & Consumo Energético & 01 & 0,7 \\
\hline & & Ambos & 02 & 1,4 \\
\hline & \multicolumn{2}{|r|}{ Sub-Total } & 32 & 22,7 \\
\hline & \multicolumn{2}{|r|}{ Total } & 141 & 100,0 \\
\hline
\end{tabular}

* Percentual do total dos estudos encontrados $(n=141)$ 
parte da revisão, adotaram-se alguns critérios. Um dos critérios foi o estudo citar em seu título ou em seu resumo a utilização de métodos para determinar o gasto energético e/ou o consumo energético diário total de forma quantitativa, isto é, em medidas como calorias ou joules. Outro critério adotado foi que o estudo tivesse base epidemiológica, podendo ser de caráter clínico ou de caráter populacional. Estudos de validação foram excluídos, assim como artigos de revisão.

Os resultados dos estudos que satisfizeram os critérios adotados são encontrados nos quadros 1 e 2. Desses estudos, foram obtidos 10 artigos na íntegra. A pesquisa para a obtenção dos artigos foi realizada em computadores da Universidade Federal de Santa Catarina, que estão ligados em rede e que disponibilizam os artigos que constam nas revistas nacionais e internacionais pelo sistema de periódicos da CAPES. Os demais artigos elegíveis e que não constam na revisão não estavam disponibilizados na base de dados pesquisada, nem mesmo puderam ser obtidos pelo sistema de comutação bibliográfica - COMUT. Também houve a tentativa de solicitar os artigos aos autores, via email, a qual não teve êxito.

Quadro 2. Quantidade de artigos localizados, não elegíveis, elegíveis e obtidos para a revisão sistemática.

\begin{tabular}{|l|c|}
\hline & MEDLINE (n) \\
\hline Artigos localizados & 141 \\
Artigos não-elegíveis & 109 \\
Artigos elegíveis & 32 \\
Artigos obtidos & 10 \\
Estudos epidemiológicos clínicos & 06 \\
Estudos epidemiológicos & 04 \\
populacionais & \\
\hline
\end{tabular}

\section{RESULTADOS}

Os dados de caracterização dos estudos obtidos encontram-se no Quadro 3. Nesse quadro constam dados dos autores, país e ano de publicação, tipo de estudo, design, população e amostra e o objetivo do estudo.

No Quadro 4, constam os dados relativos aos métodos de consumo e gasto energético empregados nos estudos revisados. Alguns aspectos são destacados no quadro: emprego dos métodos; citação dos estudos de validação por parte do autor; coeficientes de validação e reprodutibilidade; discussão do autor referente aos métodos utilizados na população do estudo.

\section{DISCUSSÃO}

Este trabalho teve como objetivo identificar, descrever e analisar os métodos de determinação do gasto e consumo energéticos em estudos epidemiológicos clínicos e populacionais. A população de referência foi de crianças e adolescentes na idade de 6 a 18 anos. Desses trabalhos, 06 foram de epidemiologia clínica e 04 de epidemiologia populacional. Para melhor compreensão do texto, a discussão foi dividida em duas partes: métodos de determinação do gasto energético e métodos de determinação do consumo energético.
Métodos de determinação do gasto energético

Nos estudos de epidemiologia clínica, os métodos de determinação do gasto energético foram água duplamente marcada (03 estudos ${ }^{12,13,21}$ ) e calorimetria indireta (04 estudos $\left.{ }^{12,13,20,21}\right)$. A técnica de água duplamente marcada é considerada o padrão ouro das medidas de gasto energético. É utilizada para a medida de gasto energético total, e combinada com a medida de calorimetria indireta (medindo a taxa metabólica basal) ela pode ser utilizada para determinar o gasto energético de atividade física.

A técnica de água duplamente marcada (ADM) é baseada na taxa de desaparecimento de biomarcadores (isótopos estáveis) ${ }^{29}$. A estimativa do gasto energético é obtida através de equações que determinam a quantidade de $\mathrm{CO}_{2}$ expelida, e de equações clássicas de calorimetria indireta ${ }^{30}$. A medida de ADM é realizada por um período de coletas de 7 a 14 dias $^{22,23, . ~ E ́ ~}$ uma medida de gasto energético habitual, já que não é sensível a variações mensais e sazonais. Sua sensibilidade é demonstrar modificações diárias e semanais.

Apresenta nível de exatidão (validação) na ordem de 5 a $10 \%$, e de precisão (reprodutibilidade) de 3 a $5 \%$, relativo a dados de calorimetria indireta ${ }^{24,30}$. Ela tem sido validada em uma ampla quantidade de sujeitos e circunstâncias, incluindo adultos, crianças e adolescentes, sujeitos em estado de equilíbrio e desequilíbrio no balanço energético ${ }^{11,22}$.

Algumas questões valem a pena serem ressaltadas em relação ao método. Speakman ${ }^{23}$ destacou que, apesar de existirem diferentes equações para determinação da quantidade de $\mathrm{CO}_{2}$ expelida, a influência dessas equações na precisão do método é na ordem de $6 \%$, e pode ser desprezada. Apesar disso, o autor ressalta a importância de cuidados na escolha dessas equações quando o intuito é a validação de outros métodos. Dos estudos analisados que utilizaram água duplamente marcada, nenhum especifica qual equação foi utilizada.

$\mathrm{O}$ aspecto essencial em relação à precisão do método é a duração do período de realização das coletas, sendo o principal responsável pelo coeficiente de variação intra-indivíduo ${ }^{11}$. Variáveis como mudança de peso e sexo não foram significativos na explicação desse coeficiente de variação. Os dados da literatura apontam um coeficiente de variação na ordem de 8 a $25 \%$, dependente desse período ${ }^{11}$.

As limitações do método da água duplamente marcada, segundo Goran ${ }^{24,29}$, são: o custo excessivo de cada medida; a necessidade de um espectrômetro de massa para analisar as amostras; o método provém uma medida direta da produção de $\mathrm{CO}_{2}$, e não de gasto energético; não demonstra o padrão das atividades realizadas pelo indivíduo, pois o método fornece um valor de gasto energético total por um dado período; o método não é efetivo para estudos epidemiológicos.

A praticidade do método de água duplamente marcada na medição do gasto energético total acaba sendo um grande atrativo, quando comparado ao método de calorimetria indireta. A necessidade de 
Quadro 3. Estudos epidemiológicos que investigaram o consumo e o gasto energético de crianças e/ou adolescentes.

\begin{tabular}{|c|c|c|c|}
\hline Autor/País/Ano & Tipo de estudo & Design & População e Amostra \\
\hline $\begin{array}{l}\text { Arpadi et al. }{ }^{12} \\
\text { Estados Unidos, } \\
2000\end{array}$ & $\begin{array}{l}\text { Epidemiológico } \\
\text { clínico. }\end{array}$ & $\begin{array}{l}\text { Analítico transver- } \\
\text { sal }\end{array}$ & $\begin{array}{l}\text { Crianças infectadas com HIV. } \\
16 \text { crianças com deficiência no } \\
\text { crescimento ( } 8.3 \pm 2.4 \text { anos) e } 26 \\
\text { crianças com crescimento normal } \\
\text { ( } 6.5 \pm 1.8 \text { anos), }\end{array}$ \\
\hline
\end{tabular}

Bandini et al. ${ }^{13}$

Estados Unidos, 2004

Epidemiológico clínico.

Analítico longitudinal.

Analítico transversal.

Gray e Smith ${ }^{14}$,
Estados Unidos, 2003 clínico.

Epidemiológico populacional

Estados Unidos, 2005

Stockman et al. ${ }^{16}$ Canadá, 2004

Rennie et al. ${ }^{17}$

Inglaterra,

Grã-Bretanha, 2005

\begin{abstract}
populacional.
\end{abstract}
Epidemiológico

Epidemiológico clínico

sal.

Estudo prospectivo de coorte.

nalítico transver-

180 adolescentes, de 14 a 18 anos, saudáveis, escolares e de grupos comunitários.

1152 meninas negras e 1135 brancas acompanhadas dos 9 ou 10 anos até os 18 ou 19 anos. Amostra proveniente do estudo do NGHS.

155 jovens americanos natianos).

Estudo 1 - escolares de famílias de menor poder aquisitivo. 2092 crianças de 10-11 anos e 1266 adolescentes de 14-15 anos.

Estudo 2 - amostra representativa da população. 2127 jovens de 4 a 18 anos

152 lares de duas vilas da zona rural, totalizando 304 lares, jovens de 3 a 18 anos $(n=519)$

Epidemiológico populacional

Estudo prospectivo de coorte.

Tetens et al.
Bangladesh, 2003

Tur et al. ${ }^{19}$

Palma de Maiorca

Epidemiológico

Analítico transverpopulacional sal,

Estudo representativo dos escolares de 14 a 18 anos, de escolas públicas e particulares $(n=445)$

Laessle el al. ${ }^{20}$

Epidemiológico Alemanha, 1997
Descritivo trans-

versal

25 crianças obesas e 21 não obesas, com idade de 8 a 12 anos.

Objetivo do estudo

Verificar as associações entre a replicação do HIV, balanço energético, composição corporal e crescimento em crianças com HIV - associado a deficiências no crescimento.

Testar a hipótese de que o baixo gasto energético durante o período pré-menarca constitui um fator de risco para o aumento no peso e no percentual de gordura durante a adolescência.

Investigar a associação entre condicionamento físico, nível de atividade física, ingestão energética e IMC de uma população de jovens nativos americanos.

Examinar a relação longitudinal de mudanças na atividade física habitual e mudanças no IMC e adiposidade

Determinar e comparar a distribuição energética e de nutrientes entre refeições e lanches, bem como relacionar a quantidade de episódios alimentares com o IMC e com a ingestão energética de meninos adolescentes.

Verificar o percentual de subrelato do consumo energético em dados coletados no National Diet and Nutrition Survey (NDNS) da população jovem de 1997 e o Diets of British School Children (DBSC) pesquisa em 1983 pesquisas.

Testar a hipótese de que as pessoas adaptam similarmente o seu consumo energético às modificações sazonais, sem ocorrer diferenças entre as idades e os sexos.

Descrever e avaliar as associações entre fatores sócio-demográficos, estilo de vida e qualidade da dieta entre adolescentes de Palma de Maiorca

Comparar taxa metabólica de repouso, consumo alimentar, insulina e hormônio do crescimento entre crianças obesas e não obesas. 
Quadro 4. Descrição dos métodos para a determinação do consumo e do gasto energético empregados nos estudos epidemiológicos revisados.

\begin{tabular}{|c|c|c|c|}
\hline \multirow[b]{2}{*}{ Estudos } & \multicolumn{3}{|l|}{ Descrição dos Métodos Empregados } \\
\hline & Consumo Energético (CoE) & Gasto Energético (Ge) & Validação \\
\hline $\begin{array}{l}\text { Arpadi et al. }{ }^{12} \\
(2000)\end{array}$ & Múltiplos Recordatórios 24h. & $\begin{array}{l}\text { Taxa Metabólica de Repouso } \\
\text { (TMR) - calorimetria indireta; } \\
\text { Ge total (GeT) - água duplamente } \\
\text { marcada (ADM) por um período } \\
\text { de } 10 \text { dias. }\end{array}$ & $\begin{array}{l}\text { O autor não cita nenhum estudo de } \\
\text { validação para a medida do CoE. }\end{array}$ \\
\hline $\begin{array}{l}\text { Bandini et al. }{ }^{13} \\
(2004)\end{array}$ & $\begin{array}{l}\text { Questionário de freqüência alimen- } \\
\text { tar semiquantitativo }\end{array}$ & $\begin{array}{l}\text { TMR - equações de Weir (calori- } \\
\text { metria indireta) } \\
\text { GeT - água duplamente marcada; } \\
\text { Gasto com atividade física - equa- } \\
\text { ção: }(0,9 \times \text { GeT) - TMR }\end{array}$ & $\begin{array}{l}\text { O questionário utilizado para deter- } \\
\text { minar o CoE foi validado somente } \\
\text { para adultos }\end{array}$ \\
\hline $\begin{array}{l}\text { Gray e Smith }{ }^{14} \\
(2003)\end{array}$ & Recordatório 24 horas & Não determinou & $\begin{array}{l}\text { O estudo não apresentou validação } \\
\text { para a sua população. }\end{array}$ \\
\hline $\begin{array}{l}\text { Kimm } \\
(2005)\end{array}$ el al. ${ }^{15}$ & $\begin{array}{l}\text { Registro dietético de } 3 \text { dias. Revisa- } \\
\text { do com os participantes através de } \\
\text { nutricionistas treinados. O CoE foi } \\
\text { obtido em Kcal/dia como a média } \\
\text { dos } 3 \text { dias }\end{array}$ & $\begin{array}{l}\text { Questionário de atividade física } \\
\text { habitual (HAQ) adaptado. Relato } \\
\text { das atividades nos últimos } 12 \\
\text { meses. O escore obtido (MET- } \\
\text { vezes/semana) foi o somatório do } \\
\text { escore de cada atividade relatada. } \\
\text { Escore da atividade = MET x fre- } \\
\text { qüência semanal } x \text { fração do ano } \\
\text { de realização da atividade (maioria } \\
\text { do ano }=1 ; \text { metade }=0,5 \text {; pequena } \\
\text { parte }=0,25 \text { ). }\end{array}$ & $\begin{array}{l}\text { HAQ validado. Houve correlação } \\
\text { fraca com a contagem do acele- } \\
\text { rômetro Caltrac }\left(r_{s}=0,09, p<0,02\right) \text {. } \\
\text { Ao longo de } 3 \text { anos, o questionário } \\
\text { demonstrou ser sensível frente às } \\
\text { mudanças na contagem do ace- } \\
\text { lerômetro (redução de } 22 \% \text { nas } \\
\text { variáveis obtidas por ambos os } \\
\text { métodos). Em relação ao registro } \\
\text { dietético, o artigo cita o estudo de } \\
\text { validação. }\end{array}$ \\
\hline $\begin{array}{l}\text { Stockman et al. }{ }^{16} \\
(2005)\end{array}$ & Registro alimentar de 3 dias & Não determinou & $\begin{array}{l}\text { O estudo não cita a validação } \\
\text { do registro alimentar para a sua } \\
\text { população. }\end{array}$ \\
\hline $\begin{array}{l}\text { Rennie et al. }{ }^{17} \\
(2005)\end{array}$ & $\begin{array}{l}\text { Registro dietético de } 7 \text { dias por } \\
\text { pesagem. }\end{array}$ & Não determinou & $\begin{array}{l}\text { A validação do registro dietético } \\
\text { foi realizada através de equações } \\
\text { que estimam as necessidades } \\
\text { energéticas. }\end{array}$ \\
\hline $\begin{array}{l}\text { Tetens et al. }{ }^{18} \\
(2003)\end{array}$ & $\begin{array}{l}\text { Método de pesagem de alimento } \\
\text { por } 24 \text { horas. }\end{array}$ & $\begin{array}{l}\text { Taxa metabólica basal-equações } \\
\text { - só empregadas dos } 18 \text { anos em } \\
\text { diante. }\end{array}$ & $\begin{array}{l}\text { O estudo não cita se os métodos } \\
\text { são validados para as condições } \\
\text { do estudo. }\end{array}$ \\
\hline Tur et al..$^{19}(2004)$ & $\begin{array}{l}\text { Questionário de Freqüência Alimen- } \\
\text { tar Semi-quantitativo }\end{array}$ & $\begin{array}{l}\text { Ge de atividade- questionário de } \\
\text { atividade física de lazer. } \\
\text { O escore de atividade física: média } \\
\text { semanal de tempo gasto x MET, } \\
\text { para cada atividade. } \\
\text { TMB - equações para adolescen- } \\
\text { tes europeus baseadas no peso, } \\
\text { altura, idade e sexo. }\end{array}$ & $\begin{array}{l}\text { O questionário de CoE foi anterior- } \\
\text { mente validado. O autor não fala se } \\
\text { o método de determinação do Ge } \\
\text { foi validado. No entanto ele cita } \\
\text { referências de emprego do método } \\
\text { em outros estudos. }\end{array}$ \\
\hline $\begin{array}{l}\text { Laessle el al. }{ }^{20} \\
(1997)\end{array}$ & $\begin{array}{l}\text { Registro dietético de } 7 \text { dias conse- } \\
\text { cutivos. . }\end{array}$ & $\begin{array}{l}\text { TMR - calorimetria indireta durante } \\
25 \text { minutos. }\end{array}$ & $\begin{array}{l}\text { O estudo destaca o cuidado que se } \\
\text { deve ter em interpretar os dados } \\
\text { do CoE para indivíduos obesos, } \\
\text { devido ao sub-relato Entretanto, } \\
\text { ressalta que essa tendência é } \\
\text { mais presente em adultos, não } \\
\text { em criança }\end{array}$ \\
\hline $\begin{array}{l}\text { Treuth et al. }{ }^{21} \\
(2000)\end{array}$ & Não utilizado & $\begin{array}{l}\text { TMB - calorimetria indireta } \\
\text { GeT - água duplamente marcada } \\
\text { durante } 14 \text { dias; } \\
\text { Gasto com atividade - equação: } \\
\text { GeT - (TMB + } 0.1 \times \text { GeT), onde foi } \\
\text { assumido que } 10 \% \text { do TEE foi devi- } \\
\text { do ao efeito térmico da comida. }\end{array}$ & $\begin{array}{l}\text { Nenhuma referência e discussão } \\
\text { sobre adaptações dos métodos } \\
\text { para o uso nessa idade. }\end{array}$ \\
\hline
\end{tabular}


permanecer em um calorímetro ou de usar um aparelho de consumo de oxigênio durante vários dias, inviabiliza tal método para a estimativa do gasto energético total.

A calorimetria indireta se baseia na relação entre gasto energético da oxidação de diferentes substratos com o consumo de oxigênio e liberação de gás carbônico. A forma mais comumente usada na literatura é a de relação de $5 \mathrm{Kcal}$ para cada litro de oxigênio consumido. Outras formas levam em consideração ainda a razão de troca respiratória (relação de liberação de gás carbônico/consumo de oxigênio), cuja função é determinar o percentual de substrato oxidado; ou ainda equações que levam em consideração tanto o consumo de oxigênio quanto a liberação de gás carbônico ${ }^{22}$.

A calorimetria é mais utilizada em situações nas quais o objetivo é medir a taxa metabólica de repouso ou basal. A diferença entre essas duas medidas está no fato de que, na basal, existe a necessidade do indivíduo estar em jejum de 10 a 12 horas, realizando uma quantidade mínima de movimento ${ }^{21}$. Essa situação pode ser facilmente encontrada logo após o sono. Haveria, portanto, a necessidade da pessoa dormir num calorímetro. Já na taxa metabólica de repouso, não há a necessidade do jejum e do pouco movimento. Goran ${ }^{22}$ encontrou que a taxa metabólica de repouso é, aproximadamente, $11 \%$ maior que a taxa metabólica basal. Portanto, haveria a necessidade de redução desses $11 \%$ no valor da taxa metabólica de repouso.

Dos estudos revisados, 02 utilizaram medida de taxa metabólica basal'18,19,21. Entre os que realizaram a medida de taxa metabólica de repouso ${ }^{12,13,20}$, nenhum fez a redução necessária. Além disso, somente o estudo de Bandini et al..$^{13}$ especificou que a forma de determinação da taxa metabólica basal foi por meio da equação de Weir (1949), que leva em consideração tanto o consumo de oxigênio quanto à liberação de gás carbônico.

A dificuldade em realizar medida de taxa metabólica basal em crianças se dá pelo fato da necessidade de dormir no calorímetro e da manutenção da pouca quantidade de movimento. Aspectos como permitir às crianças assistirem televisão pode ser uma estratégia para acalmá-las ${ }^{24}$.

Houve ainda 2 estudos ${ }^{18,19}$ que estimaram a taxa metabólica basal através de equações.

A taxa metabólica basal é o principal componente do gasto energético diário, podendo representar de 50 a $70 \%$ do total da energia gasta diariamente. Baseados nessa evidência, a FAO/WHO/UNU, a partir de 1985, passaram a nortear as recomendações energéticas com base no gasto energético diário, e sugeriram o uso de equações de predição para estimar a TMB, tanto em nível individual, quanto em nível populacional.

$\mathrm{Na}$ literatura, são amplas as equações sugeridas para estimar a TMB. As equações mais empregadas e difundidas pela literatura são as de Harris e Benedict (1919), Schofield (1985), FAO/WHO/UNU (1985) e Henry e Rees (1991). Segundo Wahrlich e Dos Anjos $^{25}$, existem mais dados para homens do que para mulheres; os coeficientes de correlação são maiores para homens, o que denota que as equações da
TMB são menos exatas em mulheres; e em crianças e adolescentes, a estimativa da TMB através de equações é mais exata do que em adultos.

Dos estudos revisados, Tetens et al. ${ }^{18}$ utilizaram as equações da $\mathrm{FAO} / \mathrm{WHO} / \mathrm{UNU}$ (1985) para predizer a TMB. Entretanto, eles utilizaram-nas somente em indivíduos acima dos 18 anos.

Já Tur et al. ${ }^{19}$ utilizaram equações específicas para a sua população de estudo, mas com o objetivo de identificar sub-relato ou supra-relato.

Para a determinação do gasto energético em atividade física, 2 estudos clínicos ${ }^{13,21}$ utilizaram equações derivadas das medidas de taxa metabólica basal e de repouso e gasto energético total. Ambos os estudos levaram em consideração um efeito térmico dos alimentos de $10 \%$ no gasto energético total. Entretanto, o estudo de Bandini et al. ${ }^{13}$ não levou em consideração a redução de $11 \%$ na taxa metabólica de repouso. Isso pode ter enviesado suas análises do gasto energético em atividade (tendência de subestimativa), já que a estimativa da mesma levou em consideração o gasto energético total e a taxa metabólica de repouso, diferente do estudo de Treuth et al. ${ }^{21}$, que para determinar $\mathrm{o}$ gasto energético em atividade levou em consideração o gasto energético total e a taxa metabólica basal.

Já nos estudos de epidemiologia populacional, nenhum estudo utilizou monitoramento da freqüência cardíaca e de acelerômetro; e dois estudos utilizaram questionário de atividade física ${ }^{15,19}$.

De acordo com Kimm et $\mathrm{a}^{26}$, o questionário e o diário de atividade física são utilizados amplamente em estudos populacionais. O questionário apresenta a vantagem de determinar a atividade física habitual; sua limitação, contudo, é a dependência da memória do indivíduo. Já o diário, apesar de não depender da memória do indivíduo, não é capaz de determinar a atividade física habitual, já que é administrado por um período específico de tempo.

Algumas questões têm sido discutidas na literatura frente aos questionários de atividade física em crianças e adolescentes. Há carência ainda de questionários validados para essa população; há a dificuldade da descrição de atividades que reflitam a realidade da criança; e há o problema de aspectos de entendimento e memória por parte das crianças ${ }^{24}$.

Dos dois estudos analisados, o estudo de Kimm et al. ${ }^{15}$ apresentou dados de validação do questionário. Em comparação com outros questionários, o nível de correlação foi baixo $\left(0,09\right.$ versus $\left.0,8^{15}\right)$. Não houve estudo de reprodutibilidade do questionário. Já o estudo de Tur et al. ${ }^{19}$, não demonstrou nenhum dado de validação.

Algo a ser analisado nesses questionários é a questão do equivalente metabólico (MET) para as atividades. De acordo com Goran ${ }^{24}$, os seguintes pressupostos devem ser considerados quando da utilização do valor MET: a intensidade do exercício pode ser explicada pelo valor MET; o custo energético da atividade é proporcional ao peso corporal; a intensidade é constante entre os indivíduos; a intensidade é constante 
para cada indivíduo em diferentes atividades; e o gasto energético na atividade é constante naquele tempo.

\section{Métodos de determinação do consumo energético \\ O consumo alimentar tem sido avaliado por} diferentes métodos com o intuito de se obter valores relativos à qualidade dos alimentos consumidos, bem como valores correspondentes às quantidades de calorias consumidas pelo indivíduo.

São diversos os métodos para medir o consumo alimentar e os fatores que interferem na precisão, validade e reprodutibilidade das informações obtidas. O entendimento dos métodos é de fundamental importância para se determinar qual deve ser empregado no sentido de possibilitar inferências sobre a ingestão alimentar individual ou de coletividades.

A dieta habitual pode ser definida como a média do consumo alimentar em um período de tempo determinado (meses ou anos), em que o indivíduo mantém um padrão constante de alimentação (métodos retrospectivos). Já a dieta atual refere-se à média de consumo alimentar em curto período de tempo corrente (métodos prospectivos) ${ }^{27}$.

Os métodos utilizados nos estudos aqui revisados foram o Recordatório de 24 horas $^{14}$, e Múltiplos Recordatórios de 24 horas $^{12}$; o Registro Alimentar de 3 dias $^{15,16}$; Registro Alimentar de 7 dias por pesagem ${ }^{17}$, e por estimativa de porções ${ }^{20}$; Registro Alimentar de 24 horas por pesagem ${ }^{18}$; e o Questionário de Freqüência Alimentar semi-quantitativo ${ }^{13,19}$.

O recordatório de 24 horas consiste numa entrevista estruturada no qual são realizadas perguntas à criança ou aos responsáveis sobre todas as coisas que ela comeu ou bebeu durante um período específico, tipicamente no dia anterior. O método é uma estimativa da ingestão atual e disponibiliza informações detalhadas sobre a alimentação do indivíduo. É muito utilizado para inferências sobre a ingestão em grupos populacionais. Devido às variações intra-individuais, são necessários múltiplos recordatórios de 24 horas para estimar com exatidão a ingestão habitual de nutrientes ${ }^{27}$.

No estudo de Gray e Smith ${ }^{14}$, foi utilizado o Recordatório de 24 horas somente com as crianças acima dos 9 anos de idade, através de uma entrevista direcionada às próprias crianças do estudo. $\mathrm{O}$ autor relata que pode ter ocorrido sub-relato da ingestão pelo fato dos jovens não entenderem o tamanho das porções, não lembrarem o que comeram, ou não registrarem as quantidades certas de alimentos consumidos.

No estudo de Arpadi et $\mathrm{al}^{12}$, os autores relatam que um único pesquisador realizou uma entrevista semi-estruturada usando modelos de alimentos. Os autores não padronizam a quantidade de vezes que ele utiliza o recordatório de 24 horas, nem mesmo cita a quem foi direcionada a entrevista realizada. Os autores só relatam que a média diária da ingestão de cada criança foi comparada com as recomendações energéticas para a idade. Esta falta de detalhamento do método denota sérios problemas para realizar inferências sobre o correto emprego do mesmo, haja vista a idade das crianças avaliadas nesse estudo (aproximadamente 6-8 anos).

McPherson et al. ${ }^{28}$ enfatizam que para crianças menores de 9 anos, é necessário o auxílio dos pais ou de pessoas responsáveis pela sua alimentação na utilização do recordatório. Para os autores, as vantagens do método são: poder ser administrado em um curto tempo; a definição precisa do tempo de recordação da ingestão alimentar; a ingestão pode ser quantificada; o procedimento não altera a ingestão habitual do indivíduo. Além disso, a baixa exigência do avaliado, a facilidade de poder ser realizado por telefone, e do procedimento poder ser automatizado são outras vantagens inerentes ao método. Como desvantagens do método são citadas: o fato do recordatório depender da memória do indivíduo, a dificuldade em estimar o tamanho das porções (notável em crianças), a necessidade de entrevistador treinado e o custo para coletar e codificar.

A validação do instrumento com um método padrão demonstrou percentuais de discordância da ordem de 34 a $18 \%{ }^{28}$.

O Registro alimentar provê informações sobre a ingestão atual de um indivíduo ou grupo populacional. Nesse método, o indivíduo ou a pessoa responsável (caso de crianças) anota em formulários específicos, todos os alimentos consumidos ao longo de um ou mais dias, devendo também anotar os alimentos consumidos fora do lar. Normalmente, o método é aplicado por 3, 5 ou 7 dias. A aplicação do método deve ser em dias alternados, e abranger um dia do fim de semana. $O$ registro pode ser aplicado de duas maneiras: na primeira o indivíduo deve registrar o tamanho da porção consumida com base em medidas caseiras ou com medidas previamente estabelecidas; e na segunda, todos os alimentos devem ser pesados e registrados, tanto os consumidos quanto os alimentos jogados fora. Em ambos os casos deve haver um detalhamento da forma de preparo do alimento, bem como dos ingredientes que o compõe ${ }^{11}$.

Dos estudos analisados, 5 utilizaram o método de registro alimentar. Kim et al. ${ }^{15}$ e Stockman et al. ${ }^{16}$, utilizaram o registro alimentar de 3 dias com base no registro das porções consumidas por parte dos sujeitos do estudo.

O Registro alimentar de 7 dias foi utilizado em dois estudos. No estudo de Rennie et al. ${ }^{17}$ foi utilizado o registro dietético por pesagem; os alimentos consumidos fora de casa eram registrados em um diário separado ou numa caderneta. Os alimentos consumidos na escola tinham sua composição e tamanho da porção coletados na escola através de balanças e livros de registro disponíveis nas cantinas escolares. Nesse estudo, houve sub-relato do método de registro dietético, não havendo diferença entre os gêneros e subestimando as necessidades energéticas em média $21 \%$ para meninos e $20 \%$ para as meninas. Houve uma tendência de aumento com a idade (até $34 \%$ ) e com a prevalência de sobrepeso (até 139\%). No estudo de Laessle et al. ${ }^{20}$, as porções foram medidas através de uma balança caseira e também 
pela estimativa do tamanho da porção quando a escala não pôde ser utilizada. Os autores também discutiram a possibilidade de poder ter ocorrido o sub-relato, mas eles argumentam que essa não é uma tendência encontrada em estudos com crianças.

Um estudo utilizou o Registro Alimentar de 24 horas por pesagem (Tetens et al. ${ }^{18}$ ). Todos os alimentos consumidos no lar eram pesados por um pesquisador treinado que ficava na casa. Os alimentos foram convertidos em alimentos crus antes de o valor energético ser computado, com o uso de tabelas de composição nutricional de alimentos de Bangladesh. Os autores desse estudo justificaram o emprego do método para estudos sazonais.

Como vantagens desse método, destacam-se: o registro não depende da memória do indivíduo, é definido por um período de registro, a ingestão pode ser quantificada, o treinamento e as padronizações de como realizar o registro podem ser realizadas em grupo, e os procedimentos podem ser automatizados. Como desvantagens pode-se citar a necessidade da criança ou sujeito da pesquisa ser alfabetizado, e existir uma alta exigência imposta pelo registro para o sujeito avaliado; os alimentos preparados em casa nem sempre são corretamente registrados; os registros, principalmente por pesagem, podem levar o indivíduo a alterar seus hábitos alimentares; e o método também é considerado custoso para coletar e codificar os dados ${ }^{28}$.

Os questionários de freqüência alimentar, que provêem uma medida usual da ingestão alimentar, são muitas vezes utilizados em estudos epidemiológicos pela sua facilidade de administração, seu menor custo e a fácil adaptação para estudos populacionais. Os questionários podem ser classificados como qualitativos, semiquantitativos e quantitativos, e possuem basicamente dois componentes: uma lista de alimentos e um espaço onde o indivíduo responderá com que freqüência ele consome este alimento. É importante ressaltar que o pesquisador precisa muitas vezes adaptar a lista de alimentos para a população do estudo. O período sobre o qual a pessoa vai remeter a sua ingestão é definido pelo pesquisador: pode ser relativo há um ano (passado), mês, semana ou até a dias anteriores. O questionário pode ser autoadministrado ou conduzido com assistência de forma individual ou em grupo ${ }^{27}$.

Dos estudos revisados, dois utilizaram Questionário de Freqüência Alimentar Semiquantitativo.

Bandini et al. ${ }^{13}$ projetaram um questionário autoadministrado para jovens de 9 a 18 anos. Os autores citam que o mesmo foi validado somente para adultos. Não houve o relato do período sobre o qual os jovens deveriam relatar sua freqüência alimentar.

Tur et al. ${ }^{19}$ determinaram a ingestão alimentar do último ano, tanto em nível de consumo energético quanto a ingestão de macro e micro nutrientes. A tendência de sub e supra-relato foi expressa pela razão CoE/taxa metabólica basal (TMB). Utilizaram-se os pontos de corte estabelecidos por Goldberg para excluir os sub e supra-relatos. Os autores citaram o estudo de validação do método.
Algumas considerações também podem ser feitas quanto ao emprego desse método para jovens. Como vantagens McPherson et al. ${ }^{28}$ relatam que não há necessidade de entrevistadores treinados e as entrevistas podem ser auto-administradas; o método tem um custo de coleta relativamente menor; a exigência por parte dos avaliados é baixa; a dieta total, ou alguns alimentos ou nutrientes específicos podem ser avaliados; pode ser usado para classificar quanto à ingestão de nutrientes; e o procedimento pode ser automatizado. Como desvantagens o questionário de freqüência alimentar depende da memória do indivíduo, o período do registro pode ser impreciso e a quantificação pode ser muito pobre por problemas de imprecisão na utilização de padrões de estimativa de tamanhos de porções; e não são obtidas descrições específicas do alimento e formas de preparação ${ }^{28}$.

\section{CONSIDERAÇÕES FINAIS}

Nos estudos de epidemiologia clínica, os métodos de gasto energético utilizados foram os mais custosos, mas que apresentam maior eficácia na determinação do gasto energético individual. Assim, geralmente a amostra desses estudos é menor, e possíveis inferências e extrapolações para condições reais, isto é, para a população geral, não são possíveis, ou não possuem força de determinação.

Algumas considerações metodológicas são evidentes e precisam ser sempre enfatizadas no emprego desses métodos, com o intuito de diminuir a influência de possíveis vieses provenientes dos mesmos. Exemplos como o período de aplicação da técnica de água duplamente marcada, que é o que mais determina o coeficiente de variação do sujeito; a própria estimação do gasto energético em atividade, estimado através da subtração do gasto energético total e da taxa metabólica de repouso, que se não houver o fator de correção implica um viés nos resultados; a utilização das equações de estimativa da taxa metabólica basal e da taxa metabólica de repouso e as superestimações que determinadas equações podem gerar.

Em estudos epidemiológicos populacionais, os métodos de determinação do gasto energético em atividade são muito dependentes do padrão de atividade física que as pessoas demonstram, bem como da acurácia com que as pessoas conseguem relatar o que elas fazem de atividade física durante um dia.

Nos métodos de determinação do consumo alimentar, também são necessários cuidados na realização de inferências quanto aos resultados. Dessa forma, considerações devem ser feitas frente ao uso de métodos que exijam da memória do indivíduo, ou que possuam um caráter mais intrusivo. Os métodos que exigem da pessoa um dispêndio de tempo muito grande e um esforço cognitivo também devem ser conduzidos de forma a serem analisados à luz das limitações que apresentam.

Deve-se buscar detalhar o máximo possível os cuidados tidos com o emprego de todos os métodos, tanto de gasto quanto de consumo energético, para 
que os mesmos sejam passíveis de confirmação e replicação. Esse esforço é necessário no intuito de possibilitar a compreensão dos resultados e de realizar possíveis inferências a partir deles.

\section{REFERÊNCIAS BIBLIOGRÁFICAS}

1. Lobstein $T$, Baur L, Uauy R for the IASO International Obesity Task Force. Obesity in children and young people: a crisis in public health. Obes Rev 2004;5(Suppl.1):4-85.

2. Wang Y. Epidemiology of childhood obesity: methodological aspects and guidelines: What's new? Int J Obes 2004;28(Suppl 3):S21-S28.

3. Fisberg M, Baur L, Chen W, Hoppin A, Koletzko B, Lau $D$, et al. Obesity in children and adolescents. Working Group Report of the Second World Congress of Pediatric Gastroenterology, Hepatology, and Nutrition. J Pediatr Gastroenterol Nutr 2004;39:S678-S687.

4. Speakman JR. Obesity: the integrated roles of environment and genetics. J Nutr 2004;134:20902105S.

5. Loos RJF, Bouchard C. Obesity: is it a genetic disorder? J Intern Med 2003;254:401-425.

6. Stubbs RJ, Tolkamp BJ. Control of energy balance in relation to energy intake and energy expenditure in animals and man: an ecological perspective. Br J Nutr 2006;95:657-676.

7. Livingstone MBE, Robson PJ, Wallace JM, McKinley MC. How active are we? Levels of routine physical activity in children and adults. Proc Nutr Soc 2003;62(3):681-701.

8. Bratteby LE, Sandhagen B, Fan H, Enghardt H, Samuelson G. Total energy expenditure and physical activity as assessed by the doubly labeled water method in Swedish adolescents in whom energy intake was underestimated by 7-d diet records. Am J Clin Nutr 1998;67:905-911.

9. Veldhuis JD, Roemmich JN, Richmond EJ, Rogol AD, Lovejoy JC, Sheffield-Moore M, et al. Endocrine Control of body composition in infancy, childhood and puberty. Endocr Rev 2005;26:114-146.

10. Black AE. Physical activity levels from a meta-analysis of doubly labeled water studies for validating energy intake as measured by dietary assessment. Nutr Rev 1996;54(6):170-174.

11. Livingstone MBE, Black AE. Markers of the validity of reported energy intake. J Nutr 2003;133(Suppl 3):895S$920 \mathrm{~s}$.

12. Arpadi SM, Cuff PA, Kotler DP, Wang J, Bamji M, Lange $M$, et al. Growth velocity, fat-free mass and energy intake are inversely related to viral load in HIV-infected children. J Nutr 2000;130:2498-2502.

13. Bandini LG, Must A, Phillps SM, Naumova EM, Dietz $\mathrm{WH}$. Relation of body index and body fatness to energy expenditure: longitudinal changes from preadolescence through adolescence. Am J Clin Nutr 2004;80:1262-1269.

14. Gray A, Smith C. Fitness, dietary intake, and body mass index in urban Native American youth. J Am Diet Assoc 2003;103:1187-1191.

15. Kimm SYS, Glynn NW, Obarzanek E, Kriska AM, Daniels SR, Barton BA, et al. Relation between the changes in physical activity and body-mass index during adolescence: a multicentre longitudinal study. Lancet 2005;366:301-307.

16. Stockman NKA, Schenkel, TC, Brown JN, Duncan AM. Comparison of energy and nutrient intake among meals and snacks of adolescent males. Prev Med 2005;41:203-210.

17. Rennie KL, Jebb AS, Wright A, Coward WA. Secular trends in under-reporting in young people. $\mathrm{Br} \mathrm{J}$ Nutr 2005;93:241-247.

18. Tetens I, Hels O, Khan NI, Thilsted SH, Hassan N. Ricebased diets in rural Bangladesh: how do diferents age and sex groups adapt to seasonal changes in energy intake? Am J Clin Nutr 2003;78:406-413.

19. Tur JÁ, Puig MS, Benito E, Pons A. Associations between sociodemografic and lifestyle factors and dietary quality among adolescents in Palma de Mallorca. Nutrition 2004;20:502-508.

20. Laessle RG, Wurmser H, Pirke KM. A comparasion of resting metabolic rate, self-rated food intake, growt hormone, and insulin levels in obese and nonobese preadolescents. Physiol Behav 1997;61(5):725-729.

21. Treuth MS, Butte N, Wong WW. Effests of familial predisposition to obesity on energy expenditure in multiethnic prepubertal girls. Am J Clin Nutr 2000;71:893-890.

22. Black AE, Prentice AM, Goldberg GR, Jebb SA, Bingham $\mathrm{SA}$, Livingstone MB, et al. Measurements of total energy expenditure provide insights into the validity of dietary measurements of energy intake. J Am Diet Assoc 1993;93(5):572-9.

23. Speakman JR. The history and theory of the doubly labeled water technique. Am J Clin Nutr 1998;68(suppl):932S938S.

24. Goran MI. Measurements issues related to studies of childhood obesity: Assesment of body composition, body fat distribuition, physical activity, and food intake. Pediatrics 1998;101:505-518.

25. Wahrlick V, Dos Anjos LA. Aspectos históricos e metodológicos da medição e estimativa da taxa metabólica basal: uma revisão da literatura. Cad Saude Publica 2001;17(4):801-817.

26. Kimm SYS, Glynn NW, Kriska AM, Fitzgerald SL, Aaron DJ, Similo SL, et al. Longitudinal changes in physical activity in a biracial cohort during adolescence. Med Sci Sports Exerc 2000;32(8):1445-1459.

27. Fisberg RM, Martini LA, Slater B. Métodos de inquéritos alimentares. In: Fisberg RM, Slater BM, Marchioni DML; Martini,LA. Inquéritos alimentares: Métodos e bases científicos. Barueri, SP: Manole, 2005.

28. McPherson SR, Hoelscher DM, Alexander M, Scanlon KS, Serdula MK. Dietary assessment methods among school-age children: validity and reliability. Prev Med 2000;30:S11-S33.

29. Goran MI. Application of the doubly labeled water technique for studying total energy expenditure in young children: a review. Pediatr Exercise Sci 1994;6:11-30.

30. Bellisle F. The doubly-labeled water method and food intake surveys: a confrontation. Rev Nutr Campinas 2001;14(2):125-133.

\section{Endereço para Correspondência}

Deivis Elton Schilickmann Frainer

Rua dos Protestantes, n 28, apto 301, Garcia

CEP: 40100-100. Salvador, Bahia, Brasil.

E-mail: frainer_de@yahoo.com.br
Recebido em 23/02/07

Revisado em 30/05/07

Aprovado em 10/10/07 